\section{$\underset{\substack{\text { hommes } \\ \text { \& migrations }}}{ }$}

\section{Hommes \& migrations}

Revue française de référence sur les dynamiques

migratoires

$1301 \mid 2013$

Migrations et mondes ruraux

\title{
Les travailleurs agricoles migrants en Italie du Sud
}

Entre incompréhension, instrumentalisation et solidarités locales

\section{Romain Filhol}

\section{Q OpenEdition \\ 1 Journals}

\section{Édition électronique}

URL : http://journals.openedition.org/hommesmigrations/1932

DOI : 10.4000/hommesmigrations.1932

ISSN : 2262-3353

\section{Éditeur}

Musée national de l'histoire de l'immigration

\section{Édition imprimée}

Date de publication : 1 janvier 2013

Pagination : 139-147

ISBN : 978-2-919040-21-6

ISSN : 1142-852X

\section{Référence électronique}

Romain Filhol, «Les travailleurs agricoles migrants en Italie du Sud», Hommes \& migrations [En ligne], 1301 | 2013, mis en ligne le 31 décembre 2015, consulté le 10 décembre 2020. URL : http:// journals.openedition.org/hommesmigrations/1932; DOI : https://doi.org/10.4000/ hommesmigrations.1932 


\title{
LES TRAVAILLEURS \\ AGRICOLES MIGRANTS \\ EN ITALIE DU SUD
}

ENTRE INCOMPRÉHENSION, INSTRUMENTALISATION ET SOLIDARITÉS LOCALES

par ROMAIN FILHOL, doctorant en géographie, École doctorale

"Ville, transports et territoires", université Paris-Est

\author{
Dans les régions du sud de l'Italie, la tension monte \\ à l'égard des ouvriers agricoles migrants, sur fond de chômage \\ et de prétendue inutilité de ces travailleurs. Embauchés \\ de manière informelle pour exécuter des tâches que personne \\ d'autre ne veut faire, les migrants doivent subir l'intolérance \\ de la population. Une aubaine pour de nombreux exploitants \\ agricoles qui tentent de faire de cette main-d'œuvre corvéable \\ à merci un modèle pour le travail agricole.
}

Coupant court aux explications politico-culturelles mentionnées, cet article entend montrer à travers une étude de la filière agro-alimentaire que les conflits dont il est ici question ne sont que la partie émergée de toute une mécanique d'instrumentalisation des migrants.

Vu les échos dans les médias, il est aujourd'hui difficile de parler des travailleurs agricoles migrants d'Italie du Sud sans évoquer l'épisode de Rosarno. Difficile, effectivement, d'oublier que cette commune de Calabre fut, en janvier 2010, le théâtre de ce que de nombreux témoignages qualifièrent de "chasse à l'Africain" à l'encontre des braccianti, les ouvriers agricoles - pour la plupart étrangers - chargés de récolter les agrumes de la plaine de Gioia Tauro ${ }^{1}$. Ces derniers, dans un espace fortement investi par la "Ndrangheta (mafia calabraise), osèrent se révolter contre l'agression de l'un des leurs, et plus généralement contre les conditions de travail indignes auxquelles ils sont soumis : journées de travail trop longues et trop peu rémunérées (environ une vingtaine d'euros pour une journée de dix heures), absence de contrat de travail, précarité de l'emploi, racisme ambiant et absence de structures d'accueil, ce qui les contraints à dormir dans des bâtiments abandonnés². Or cette "révolte" se solda par la mise en place de "rondes" qui, chargées d'éloigner et de passer à tabac les migrants trahis par leur couleur de peau, ne furent pas sans rappeler les heures sombres de l'Italie fasciste. Elles effrayèrent aussi suffisamment les braccianti présents pour qu'aujourd'hui, 
nombre d'entre eux aient peur d'y retourner "faire la saison ${ }^{3 "}$. Dans une tentative d'explication des événements, on entendit deux types d'arguments. Le premier, avancé par le ministre de l'Intérieur du gouvernement Berlus-

La plupart des actes de violence infligés aux migrants trouvent leur justification dans un postulat simple mais malheureusement efficace : celui de leur "inutilité", dans un espace en proie à de nombreuses difficultés économiques et sociales. té tant au gouvernement au pouvoir qu'aux individus responsables des agressions sur les migrants, ces deux types d'arguments passaient néanmoins à côté de l'essentiel.

D'une part, ils ne prenaient pas en compte la "révolte de Rosarno", bien loin d'être un événement isolé, n'était que l'énième épisode de toute une série de révoltes mettant en scène travailleurs agricoles migrants et sociétés rurales du Sud (voir encadré). D'autre part, ils passaient sous silence deux dynamiques parallèles qui, depuis les années 1990, agitent de concert les campagnes du Mezzogiorno, à savoir une forte augmentation des flux migratoires et l'érosion constante des bénéfices issus de l'activité agricole.

Coupant court aux explications politico-culturelles mentionnées, cet article entend montrer à travers une étude de la filière agro-alimentaire que les conflits dont il est ici question ne sont que la partie émergée de toute une mécanique d'instrumentalisation des migrants qui, fondée sur l'incompréhension suscitée par une immigration considérée comme "inutile", permet aux entrepreneurs agricoles de profiter d'une main-d'œuvre à moindre coût. Nous verrons alors comment, au-delà des révoltes, l'émergence d'une solidarité locale est d'au- tant plus indispensable qu'il ne s'agit pas seulement de protéger les travailleurs migrants, mais bien l'ensemble des ouvriers agricoles de la région. Ce texte va s'appuyer sur les données issues d'une enquête de terrain longue de plusieurs mois en Campanie, réalisée dans le cadre d'un mémoire de recherche pour le compte de l'université Paris-Est. Le choix de la région de Naples fut dicté par la volonté de s'immerger dans un espace d'agriculture intensive et de migration ancienne, réunissant la majorité des traits économiques et sociaux caractéristiques du Mezzogiorno ${ }^{6}$, et empreint d'une forte conflictualité entre population locale et populations migrantes. Enfin, malgré les difficultés rencontrées, mon objectif fut de réaliser des entretiens tant avec les travailleurs migrants qu'avec les exploitants agricoles, pour mieux comprendre à la fois le rôle joué par la main-d'œuvre au sein de la production agricole et les dynamiques régissant les rapports entre ouvriers et exploitants.

\section{Une immigration incomprise}

La plupart des actes de violence infligés aux migrants trouvent leur justification dans un postulat simple mais malheureusement efficace : celui de leur "inutilité", dans un espace en proie à de nombreuses difficultés économiques et sociales. Bien que ce discours ne soit pas circonscrit au Mezzogiorno, il y trouve néanmoins une résonance singulière, offrant ici plus qu'ailleurs une sorte d'expiatoire aux tensions provoquées par la rencontre entre des structures économiques et sociales anciennes et des mutations brusques liées à l'ouverture des économies et des frontières.

L'histoire du Mezzogiorno est avant tout faite de départs et, jusqu'il y a peu, rarement d'arrivées. Ainsi, les millions de Méridionaux qui, dès la fin du XIX ${ }^{e}$ siècle, quittèrent leur village en direction du Nouveau Monde, de l'Europe du Nord ou encore 




Romain Filhol, Upec.

SOURCE : ISTAT

du nord de l'Italie, ont contribué à façonner une identité d'émigrants qui, aujourd'hui encore, structure les projets et les pratiques de nombreux habitants du Sud ${ }^{7}$. Pourtant, face à ce mouvement qui perdure - comme le montrent les différentes données documentant la "fuite" des jeunes Italiens du Sud provoquée par l'actuelle crise économique ${ }^{8}$-, le Mezzogiorno s'est en l'espace de quelques décennies imposé comme un pôle d'attraction dynamique des migrations internationales à destination de l'Europe.

Après avoir connu une lente mais continuelle progression des arrivées à partir des années 1960 - grâce aux migrants en provenance d'Afrique subsaharienne et du Maghreb -, ce n'est qu'à partir de la fin des années 1990 que la croissance de la population migrante dans le Mezzogiorno s'accélère, à la faveur de la chute du bloc de l'Est et de la saturation des débouchés "traditionnels" du Nord. En venant s'ajouter aux flux existants, les migrations en provenance d'Europe de l'Est - la carte 1 nous en montre l'importance à travers l'exemple de la Campanie - vont donc permettre aux régions du Sud de connaître, durant la dernière décennie, des taux d'accroissement de la population étrangère supérieurs à ceux des régions du Nord, tout en restant probablement sous-évalués vu l'importance de la 
composante informelle révélée par les différentes campagnes de régularisation ${ }^{9}$. En somme, la rapidité avec laquelle le Mezzogiorno est passé du statut d'espace de départ à celui d'espace d'arrivée tout en demeurant un espace d'émigration - est l'un des facteurs responsables de l'incompréhension des habitants du Sud face à une immigration qu'ils n'auraient sûrement pas imaginée quelques années auparavant.

Cette incompréhension est d'autant plus forte que cette inversion des dynamiques migratoires ne semble pas liée à la disparition des facteurs "répulsifs" qui avaient poussé les

L'agriculture intensive, habitants du Sud à émigrer. secteur d'activité majeur La pauvreté y est toujours duMezzogiorno, beaucoup plus répandue est un exemple parfait pour

illustrer l'importance de la main-d'œuvre immigrée dans l'économie du Mezzogiorno. que dans le reste du pays, touchant environ $22 \%$ des habitants des régions du Sud, contre seulement $6 \%$ des habitants du Centre et du Nord. Le chômage y est lui aussi beaucoup plus élevé. En 2010, l'Institut national de statistiques italien (Istat) estimait qu'il concernait $13 \%$ de la population, contre respectivement $7 \%$ et $6 \%$ de la population du Centre et du Nord. Pire encore, ce taux monte à plus de $30 \%$ dans la catégorie des 18-29 ans.

Les revenus disponibles des familles y restent très inférieurs à ceux du reste du pays : en 2011, l'écart avec la moyenne nationale se situe entre $+9 \%$ (19 500 euros par an) et $+15 \%$ (20 500 euros par ans) pour le Centre et le Nord, contre $-23 \%$ (13 500 euros par an) pour le Sud ${ }^{10}$.

Enfin, le Mezzogiorno doit supporter tant les coûts d'une criminalité organisée devenue acteur économique et politique incontournable ${ }^{11}$ que ceux de l'échec de la politique d'industrialisation et de modernisation du Sud menée par l'État italien jusqu'en $1985^{12}$, symbolisé par le combat des ouvriers de la Fiat à Pomigliano ou à Melfi.

\section{Une véritable demande de main-d'œuvre immigrée}

Malgré les évidentes difficultés économiques du Sud, les chercheurs ont réussi à mettre en évidence un besoin de main-d'œuvre immigrée qui contraste avec l'opinion commune selon laquelle l'emploi des étrangers s'effectue au détriment de la main-d'œuvre locale.

Pour Alessandro Cangiano et Salvatore Strozza, c'est l'inadéquation entre l'offre et la demande de travail locale - un "skill mismatch" donc - qui permet de comprendre la coexistence d'un taux de chômage élevé et d'un besoin de main-d'œuvre étrangère. Le marché du travail du Mezzogiorno serait ainsi caractérisé par une "insuffisance de type qualitatif provenant de l'augmentation particulièrement élevée du niveau d'instruction des nouvelles générations, qui pourrait mal se concilier avec les nécessités d'un système productif dans lequel une part importante de l'activité est encore concentrée dans des secteurs traditionnels comme l'agriculture, le bâtiment et le bas tertiaire". En somme, "l'économie méridionale ne serait pas caractérisée par la rareté de la maind'œuvre au sens propre, il paraîtrait plutôt qu'il existe dans ces régions une niche de demande de travail déqualifié [...] pour lequel les immigrés étrangers représentent un bassin d'offre privilégiééc".

En d'autres termes, les principaux débouchés offerts à la force de travail jeune et bien formée du Mezzogiorno sont des travaux déqualifiés (les fameux trois " $\mathrm{D}$ ", “dirty, dangerous, demanding ${ }^{14 "}$ ) qu'elle n'est pas prête à accomplir, car l'augmentation de sa formation a également augmenté ses attentes de réalisation professionnelle, "ce qui

\footnotetext{
9. Sergio Carfagna, Domenico Gabrelli, Maria Pia Sorvillo, Salvatore Strozza, “Cambiamenti di status degli immigranti in Italia : risultati di un record linkage su fonti amministrative”, in Mondi Migranti, vol. 4., 2010. 10. Données Istat, 2011. 11. Fabrizio Maccaglia, “Territoires parallèles. Pouvoir et contre pouvoir criminels dans l'Italie contemporaine”, in Colette Vallat (dir.), Autres vues d'Italie. Lecture géographique d'un territoire, Paris, L'Harmattan, 2004. 12. Jacqueline Lieutaud, “La permanence du problème du Mezzogiorno italien", in Méditerranée, tome 76, vol. 34, 1992. 13. Alessandro Cangiano, Salvatore Strozzza, "Gli immigrati extracomunitari nei mercati del lavoro italiani : alcune evidenze empiriche a livello territoriale”, in Economia e Lavoro, vol. 39, n 1, 2005. 14. Benedict Anderson, "Immigration, contrôle et construction de la précarité en Grande-Bretagne", in Alain Morice, Swanie Potot (dir.), De l'ouvrier immigré au travailleur sans papiers, Paris, Karthala, 2010.
} 
la pousse à refuser toute une série d'emplois 'humbles' dont la rétribution et le prestige social sont jugés inadéquats au parcours de formation effectué15". Se met donc en place une stratégie d"attente" d'une opportunité acceptable, fondée sur la possibilité - à la fois culturelle ${ }^{16}$ et liée au fonctionnement d'un "welfare" qui s'attache surtout à protéger le "chef de ménage17" - offerte aux jeunes de rester à la charge de leurs parents. Au contraire, "il est bon de rappeler que pour les migrants, ces mêmes emplois offrent des conditions de travail et des salaires en général de loin supérieurs à ceux du pays d'origine ${ }^{18 "}$.

Pire encore, ce mouvement est renforcé par la place de l'économie irrégulière. Alors que "l'Italie méridionale est l'une des zones appartenant à un pays développé dans laquelle le travail irrégulier est le plus répand $u^{19 "}$, le refus d'accorder aux travailleurs étrangers les mêmes droits qu'aux travailleurs locaux encourage les employeurs les moins scrupuleux à favoriser l'embauche d'une main-d'œuvre immigrée qui, plus vulnérable, est moins susceptible de protester contre les conditions de travail et les rétributions accordées.

\section{Le travailleur migrant, une aubaine pour les exploitants agricoles}

L'agriculture intensive, secteur d'activité majeur du Mezzogiorno, est un exemple parfait pour illustrer l'importance de la main-d'œuvre immigrée dans l'économie du Mezzogiorno.

Selon Jean-Pierre Berlan, "on peut définir les cultures intensives par deux critères: des besoins importants de main-d'œuvre à l'hectare, très inégalement répartis au cours de l'année $e^{20 "}$. Le caractère saisonnier de

l'agriculture intensive se traduit donc par une variation temporelle forte des besoins de main-d'œuvre de chaque exploitation dite "intensive", la plupart pratiquant le maraîchage, l'arboriculture ou la tabaculture. Le tableau ci-dessus, construit à partir d'entretiens avec les membres de trois exploitations agricoles de la province de Caserta ${ }^{21}$, nous en donne un exemple concret.

Si cette variation temporelle des besoins de maind'œuvre est une certitude, il est difficile pour les exploitants agricoles de déterminer à l'avance quelles seront les périodes concernées et quelle sera la quantité de travail requis. Ces dernières vont dépendre, entre autres, du climat, du volume produit et des prix du marché.

La présence, dans les espaces d'agriculture intensive, d'une grande quantité de main-d'œuvre est donc une condition préalable à l'activité agricole, puisque aucun exploitant n'investira dans une récolte (à travers la préparation du terrain, l'ensemencement ou le traitement des jeunes pousses) s'il n'a pas au préalable la certitude d’avoir à sa disposition 
Romain Filhol, Upec

Sources : entretiens Zongo, Marcianise,

25 mai 2011 et 26 avril 2012.

suffisamment de bras pour pouvoir la mener à terme. Mais ce n'est pas tout, car cette maind'œuvre doit être mobilisable très rapidement, afin de faire face aux aléas de la production (mûrissement accéléré, avaries, augmentation temporaire des prix des denrées agricoles). Elle doit être également plus nombreuse que le nécessaire, afin d'éviter toute escalade du coût du travail liée à un risque de pénurie de main-d'œuvre durant la récolte, et la moins chère possible. Devant l'augmentation continuelle des coûts de production (intrants, carburant, semences) et l'érosion constante des bénéfices - notamment provoquée par une concurrence accrue entre les différents bassins de production agricole du pourtour méditerranéen et la baisse des aides accordées par l'Union européenne -, le travail est la seule variable du processus de production que l'exploitant agricole peut maitriser.

Aux yeux des exploitants, l'employé idéal est donc un individu souvent au chômage et prêt à accepter de dures conditions de travail contre la plus faible rémunération possible. Pour cela, le travailleur migrant se révèle être le candidat idéal. Parce que sa volonté de mener à bien son projet migratoire est suffisamment forte pour qu'il accepte des emplois qu'il n'aurait, dans d'autres conditions, pas acceptés. Parce qu'il est très mobile et n'hésite pas à se déplacer dans l'ensemble du Mezzogiorno au rythme des saisons, comme nous le montre la carte 2.

Surtout, parce que la législation italienne sur le séjour des étrangers favorise une "précarité légale" des migrants qui ne leur laisse guère d'autre choix que celui de se plier à n'importe quelles conditions de travail. En effet, parmi les travailleurs migrants que j'ai rencontrés en Campanie - pour la plupart non issus de l'Union européenne -, très peu sont ceux qui n'ont jamais eu de papiers de séjour. La plupart ont déjà pu obtenir un permis pour motif de travail, de protection humanitaire ou d'asile politique, mais les difficultés rencontrées pour se procurer les prérequis nécessaires à son renouvellement (contrat de travail, de location, et aussi plusieurs centaines d'euros de taxes) les contraignent sans cesse à replonger dans l'irrégularité. Cette force de travail largement inoccupée, placée devant la nécessité de travailler sans en avoir le droit, correspond parfaitement aux besoins des exploitants agricoles qui, placés devant l'obligation de réduire au maximum les coûts de la main-d'œuvre, ne vont pas hésiter à employer des ouvriers "au noir", 
le plus fréquemment à l'aide d'un faux contrat de travail - n'enregistrant, par exemple, qu'une seule journée de travail dans le mois. En somme, le secteur tout entier repose sur l'usage diffus, durant les pics d'activité, d'une main-d'œuvre peu regardante car nombreuse, souvent inactive et sans droits.

\section{Les ressorts de la domination des migrants : informalité, racisme et sexisme}

Pour s'assurer sur le long terme une main-d'œuvre docile et peu coûteuse, les exploitants agricoles vont mettre à profit l'irrégularité du statut des migrants et le climat d'intolérance face à cette immigration considérée comme "inutile".

Contournant les chemins légaux, l'embauche des travailleurs irréguliers va se faire à travers des pratiques que l'on pensait disparues, comme celle de "la piazza". Z., migrant burkinabé, nous en explique le fonctionnement: "Si tu veux du travail [...] on fait ce qu'on appelle le rond-point, chaque matin à 4 heures du matin. Tu vas là-bas, si les agriculteurs viennent, on peut t'appeler pour du travail, si tu acceptes pour 30 euros, tu y vas ${ }^{22}$." De cette dernière affirmation de Z., on comprend à quel point le rapport de force entre ouvriers migrants et employeurs est disproportionné. Au second va toute la liberté de fixer le prix du travail, tandis que les premiers n'ont qu'à l'accepter. Ainsi, "le patron vient au rond-point avec sa camionnette, il demande si tu bosses pour 30 euros, si tu dis non, il va au suivant, et au suivant, jusqu'au gars qui n'a pas travaillé depuis dix, vingt jours, et qui va forcément accepter ${ }^{23}$. Autre pratique héritée de l'époque des latifundia, celle de l'embauche à travers le "caporale", intermédiaire de main-d'œuvre mettant en contact exploitants et ouvriers en échange d'une retenue sur salaire. Si le phénomène n'est jamais mentionné de façon explicite - notamment car le caporale est souvent proche de la mafia -, il est pourtant simple de lire entre les lignes, comme lorsque ce technicien d'une exploitation agricole déclare: "Disons que ce sont toujours les mêmes. Compris? Il y a un chef, il y a une personne à qui nous faisons... confiance, et lui trouve d'autres personnes à appeler et pour s'occuper des papiers pour les faire travailler ${ }^{24}$." En somme, sans aucune relation "formelle" avec l'employeur (agence pour l'emploi, contrat de travail), les ouvriers agricoles migrants sont contraints à une docilité et une productivité absolues pour espérer être réembauchés.

Par ailleurs, à cette domination liée à l'embauche informelle de main-d'œuvre migrante vient s'ajouter une double domination raciale et sexuelle, qui se traduit par deux types d'attitudes de la part des employeurs. La première est celle d'une attention toute particulière portée aux "corps" de ceux qui, par leur sexe ou leur couleur de peau, diffèrent de la "norme". Les migrants racontent comment, lors du recrutement sur la "piazza", les exploitants s'attachent à scruter leurs muscles, afin de ne sélectionner que les ouvriers les plus "forts 25 ". Les femmes, également, ne sont considérées qu’à travers leur physique ; ainsi, en pleine conversation avec l'une d'elles, l'un des techniciens de l'exploitation A. lui fait remarquer qu'elle est "moche". Au contraire, il prend ostensiblement la peine de me faire Les migrants racontent remarquer que l'une d'elles a comment, lors du une belle poitrine, cherchant à tout prix à me la faire admirer à mon tour ${ }^{26}$. Enfin, les tapes "amicales" des employeurs à leurs employé(e)s sont plus que fréquentes, et le mouvement effrayé d'un ouvrier marocain à l'approche recrutement sur la "piazza", les exploitants s'attachent à scruter leurs muscles, afin de ne sélectionner que les ouvriers les plus "forts". Les femmes, également, ne sont considérées qu'à travers leur physique. de son patron m’a confirmé qu'il ne s'agissait guère de gestes bienveillants. En somme, ouvriers migrants et ouvrières - même italiennes - ne sont, pour leurs supérieurs, que des corps à exploiter, et ces derniers ne manquent aucune occasion de le leur rappeler.

Dans le même temps, les différents acteurs du monde agricole accordent aux migrants et aux 
femmes des "spécialités" qui, rapidement, se transforment en assignations. Ainsi, pour E., "les femmes italiennes s'occupent des fraises. Beaucoup. Elles préfèrent, c'est plus un travail de femmes ${ }^{27 "}$, tandis que pour M., "l'enfilage ou le désenfilage [du tabac] sont des travaux féminins, tout comme

En somme, l'usage

d'une main-d'œuvre

irrégulière a permis de

flexibiliser et donc

de précariser l'ensemble

du marché du travail

agricole du Mezzogiorno,

contribuant à le rendre toujours plus répulsif pour

la main-d'œuvre locale. la récolte des feuilles ${ }^{28}$. Quant aux migrants, selon les propos dudirigeantd'unsyndicatd'agriculteur local, "'ést amusant, parce qu'il y a des ethnies spécifiquespourchaquetypedeculture ${ }^{29 "}$. E. confirme: "Ici, nous avons les Albanais qui récoltent le tabac, les Tunisiens et les Marocains qui récoltent les pommes de terre, les Algériens qui s'occupent des vaches, bien qu'il $y$ ait aussi les Indiens pour cela ${ }^{30}$ ". En somme, l'explication de la présence migrante dans l'agriculture est toute trouvée : c'est une explication raciale, comme l'usage du terme par E. nous le confirme: "Ici, il y a plutôt des Maghrébins ou des Sénégalais... Ce sont les races les plus présentes." Tout comme les femmes choisiraient le travail agricole car cela conviendrait mieux à leur nature et à leurs désirs - pourtant exprimés par les hommes -, les différents migrants choisiraient ce travail car ils seraient détenteurs d'une aptitude particulière qui, au final, justifie a posteriori leur place dans ce segment dévalorisé du marché du travail.

\section{Quand le sort des travailleurs du Mezzogiorno est lié à celui des travailleurs migrants}

La présence d'une main-d'œuvre corvéable à merci est, à n'en point douter, une aubaine pour les exploitants agricoles du Mezzogiorno. Mais loin de profiter à l'ensemble des membres des sociétés agricoles du Mezzogiorno, cette "aubaine" ne fait que contribuer à la dégradation générale des conditions de travail au sein du secteur agricole au pro- fit des plus gros exploitants et des actionnaires des grands groupes agro-alimentaires. Le secteur agricole nous permet de voir comment les pratiques informelles, telles que le recrutement à travers "la piazza" ou l'usage d'un "caporale", ont fini par déqualifier les pratiques officielles qui, en comparaison, apparaissent bien trop "rigides". E. nous explique par exemple comment, après que l'agence pour l'emploi a été incapable de lui envoyer immédiatement 10 ouvriers agricoles, il a fini par faire appel à une équipe de travailleurs roumains "des environs" qui lui a immédiatement récolté son champ. M., lui, nous explique comment son entreprise utilise les quotas d'entrée sur le territoire italien de travailleurs étrangers pour régulariser des ouvriers déjà employés, mais illégalement au sein de son exploitation, récompensant ainsi les ouvriers les plus productifs. En somme, l'usage d'une main-d'œuvre irrégulière a permis de flexibiliser et donc de précariser l'ensemble du marché du travail agricole du Mezzogiorno, contribuant à le rendre toujours plus répulsif pour la main-d'œuvre locale.

Par ailleurs, les "louanges" des exploitants agricoles envers leurs ouvriers ou ouvrières étrangers ne cachent pas qu'une assignation identitaire, il s'agit d'une critique, en creux, de l'ouvrier agricole italien. Ainsi, E. ne cache pas préférer les Maghrébins aux Italiens pour la récolte des patates,

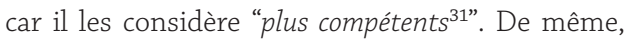
lorsqu'il me dit que la récolte des fraises est un travail de femme, il ajoute que les hommes préfèrent être au bar, à ne rien faire. En opposant la fainéantise des ouvriers agricoles italiens à la bravoure et à l'humilité des ouvrières agricoles italiennes et des ouvriers agricoles migrants, les entrepreneurs agricoles justifient le recours à une main-d'œuvre migrante et féminine par la disqualification de la main-d'œuvre masculine "locale", et avouent par là favoriser le recours à une main-d'œuvre peu protégée et plus facilement exploitable.

En somme, le destin de l'ensemble des ouvriers agricoles du Mezzogiorno - mais aussi des autres secteurs d'activités - est lié à celui des ouvriers ou 
ouvrières agricoles migrant(e)s, puisque accepter de voir ces derniers travailler pour un salaire de misère en dehors de toute protection légale, c'est ouvrir une brèche dans le droit du travail qui profite certes aux détenteurs des moyens de production, mais, plus encore, à tout un système de distribution et de commercialisation des productions alimentaires largement investi par la criminalité organisée ${ }^{32}$. La création de lien social entre migrants et population locale est donc indispensable, car elle seule peut permettre à l'ensemble des ouvriers agricoles du Sud de tenir tête aux exploitants agricoles et, au-delà, à tout un système productif fondé sur le maintien et l'exploitation d'une "armée industrielle de réserve ${ }^{33}$ ". Mais, avant tout, il est indispensable de mettre fin à la dictature du permis de séjour, ce que les syndi- cats et les centres sociaux ont bien compris à travers leur soutien concret aux procédures administratives auxquelles les migrants sont soumis. Dans ce domaine, l'action du centre social Ex-Canapificio de Caserta est particulièrement remarquable, conjuguant programme d'accueil, défense des migrants devant les administrations et les tribunaux, et actions militantes concrètes visant à créer un véritable rapport de force en faveur d'une catégorie de travailleurs marginalisés. C'est donc en toute connaissance de cause que ce texte appelle à soutenir les actions des travailleurs agricoles migrants, au nom de la défense des droits du travail et du refus d'un système productif agricole ne profitant qu'aux mafias, aux groupes agroalimentaires et à une petite poignée de grands exploitants agricoles. I

\section{Travailleurs migrants et révoltes dans le Mezzogiorno}

Un an avant la révolte de Rosarno, les travailleurs agricoles migrants de la plaine de Gioa Tauro avaient déjà organisé une marche de protestation suite à l'agression de l'un des leurs. Les premières violences publiquement reconnues à l'encontre de travailleurs agricoles migrants furent celles de Villa Literno (Campanie), le 24 août 1989, qui conduisirent à la mort du réfugié sud-africain Jerry Maslo et à la première grève de braccianti migrants. Néanmoins, l'épisode le plus sanglant fut assurément celui de Castel Volturno (Campanie également), avec l'assassinat le 18 septembre 2008 de 5 migrants africains, suivi d'une importante manifestation des travailleurs migrants originaires d'Afrique de l'Ouest de toute la région. Depuis, plusieurs grèves se sont succédé, toujours en Campanie, où les saisonniers assuraient en septembre 2010 qu'ils ne travailleraient pas pour moins de 50 euros par jour, mais aussi à Nardò, dans les Pouilles, durant l'été 2011. 